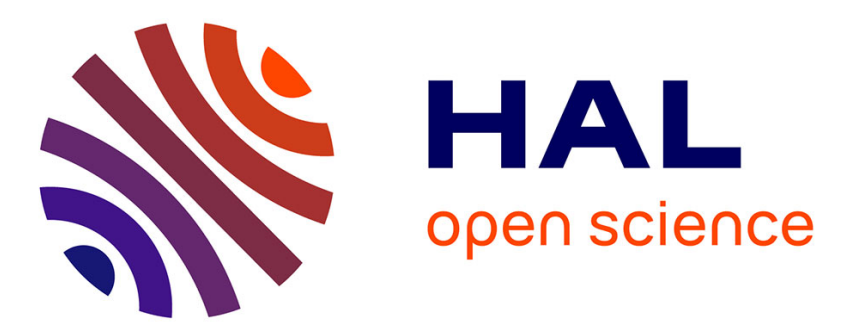

\title{
Nonparaxial analysis of continuous self-imaging gratings in oblique illumination
}

\author{
Guillaume Druart, Nicolas Guérineau, Riad Haïdar, Jérôme Primot, Pierre \\ Chavel, Jean Taboury
}

\section{- To cite this version:}

Guillaume Druart, Nicolas Guérineau, Riad Haïdar, Jérôme Primot, Pierre Chavel, et al.. Nonparaxial analysis of continuous self-imaging gratings in oblique illumination. Journal of the Optical Society of America. A Optics, Image Science, and Vision, 2007, 24 (10), pp.3379-3387. 10.1364/JOSAA.24.003379 . hal-00872939

\section{HAL Id: hal-00872939 \\ https://hal-iogs.archives-ouvertes.fr/hal-00872939}

Submitted on 14 Oct 2013

HAL is a multi-disciplinary open access archive for the deposit and dissemination of scientific research documents, whether they are published or not. The documents may come from teaching and research institutions in France or abroad, or from public or private research centers.
L'archive ouverte pluridisciplinaire HAL, est destinée au dépôt et à la diffusion de documents scientifiques de niveau recherche, publiés ou non, émanant des établissements d'enseignement et de recherche français ou étrangers, des laboratoires publics ou privés. 


\title{
Nonparaxial analysis of continuous self-imaging gratings in oblique illumination
}

\author{
Guillaume Druart, ${ }^{1, *}$ Nicolas Guérineau, ${ }^{1}$ Riad Haïdar, ${ }^{1}$ Jérôme Primot, ${ }^{1}$ Pierre Chavel, ${ }^{2}$ and Jean Taboury ${ }^{2}$ \\ ${ }^{1}$ Office National d'Etudes et de Recherches Aérospatiales, Chemin de la Hunière, 91761 Palaiseau Cedex, France \\ ${ }^{2}$ Institut d'Optique, Campus Polytechnique CD 128, 91127 Palaiseau Cedex, France \\ *Corresponding author: guillaume.druart@onera.fr
}

Received May 4, 2007; revised July 30, 2007; accepted July 31, 2007;

posted August 6, 2007 (Doc. ID 82769); published September 25, 2007

Tolerance in angles of continuously self-imaging gratings (CSIGs) is explored. The degradation in angle of the shape of the point-spread function is theoretically investigated and illustrated by simulations and experiments. The formalism presented is inspired by the one used for classical lenses and can be easily generalized to diffraction gratings. It turns out that well-designed CSIGs could be used for scanning optical systems requiring a large field of view. (c) 2007 Optical Society of America

OCIS codes: $050.0050,110.3000,110.3080$.

\section{INTRODUCTION}

During the past decade, a great number of authors have proposed various optical components for generating propagation-invariant spot arrays. These arrays can be seen as particular nondiffracting beams, as described by Durnin [1] in 1987. In practice, the most studied solution is the zero-order $\left(J_{0}\right)$ Bessel beam. For instance, the axicon introduced by McLoed [2] is a good approximation of an optical component producing a $J_{0}$ Bessel beam with a high transmittance and has been widely studied [2-7]. This optical component provides a long depth of focus with a good resolution and is a good candidate for many applications. For example, it has been integrated into scanning optical systems [8,9]. However, despite these two qualities, axicons seem to have limitations for wideangle imaging applications. Studies in [10-13] on the tolerance in angles of axicons have suggested that they are very sensitive to off-axis aberrations, but this needs further investigation. The purpose of this paper is indeed to study the tolerance angle of a particular class of optical components called continuously self-imaging gratings (CSIGs) $[14,15]$. These components have similarities with axicons in terms of depth of focus and resolution. When illuminated by a plane wave, CSIGs produce a field whose intensity profile is a propagation- and wavelengthinvariant biperiodic array of bright spots. At present, these properties are being used in the field of optical metrology, where CSIGs are used to generate reference intensity patterns made of bright spots for the control of wavefront [16] or for the measurement of the detector's spatial response [17]. In this paper, we will demonstrate that well-designed CSIGs can be relatively robust in the field in compensation for a trade-off with resolution. CSIGs can be thus adapted for the design of optical systems used for imaging purpose requiring a large field of view (FOV) and a large depth of focus.

In Section 2, general principles of CSIGs for the non- paraxial study will be presented. In Section 3, the main off-axis aberration terms will be determined. This theoretical study is inspired by the formalism used for classical lenses. In Section 4, tolerance angle criteria will be developed to assess the tolerance angle of CSIGs, and relations between phase and intensity degradation will be explored. The tolerance angle of a CSIG is the angle above which the point-spread function (PSF) is degraded by offaxis aberrations. Results from simulations will illustrate the theoretical approach. And finally, in Section 5, the degradation of the PSF will be studied experimentally.

\section{FUNDAMENTAL PROPERTIES OF CSIGs IN NORMAL INCIDENCE}

Consider, in the Fourier space, a Cartesian grid of pitch $1 / a_{0}$ and a circle (Fig. 1). The intersection of the horizontal and vertical grid lines with the circle form a set of points that can be called "orders" (see [15]), as they constitute the set of orders of any bidimensional grating of pitch $a_{0}$ in both $x$ and $y$ directions. The different orders selected have the following coordinates in the spatialfrequency domain:

$$
\begin{gathered}
\nu=\frac{p}{a_{0}}=\frac{\eta}{a_{0}} \cos (\varphi), \\
\mu=\frac{q}{a_{0}}=\frac{\eta}{a_{0}} \sin (\varphi),
\end{gathered}
$$

where $\eta / a_{0}$ is the radius of the selecting circle, and $\eta$ is thus a dimensionless value given by

$$
\eta=\sqrt{p^{2}+q^{2}} .
$$

We call CSIG any transmittance $t(x, y)$ whose spatialfrequency spectrum is the subset of those orders lying on a given circle centered at the origin. The transmittance of 


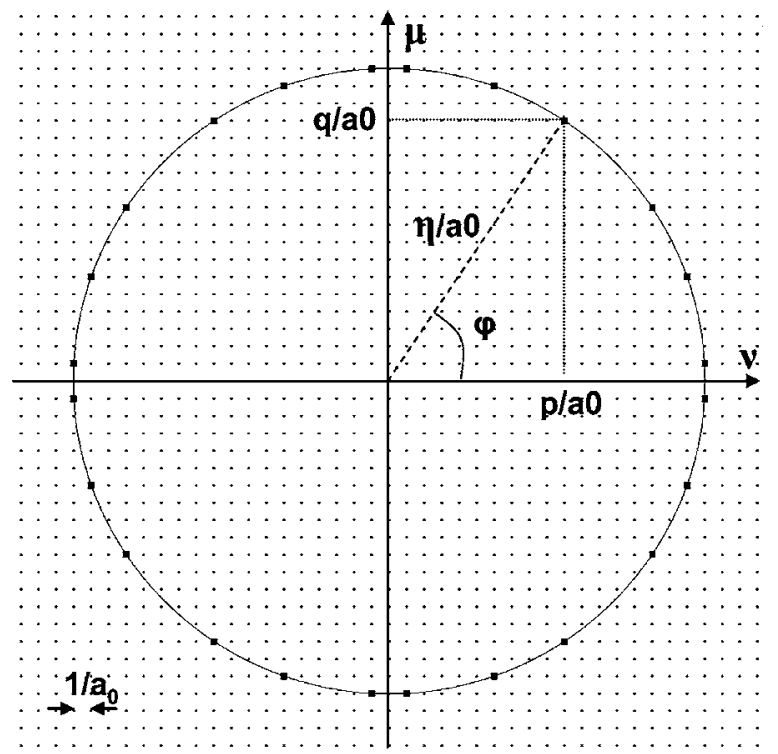

Fig. 1. Construction in the Fourier plane of a 24-order CSIG by selecting orders from a Cartesian grid with a ring of radius $\eta$.

the CSIG is given by the following relation:

$$
t(x, y)=\sum_{p, q} C_{p q} \exp \left(\frac{2 i \pi(p x+q y)}{a_{0}}\right),
$$

where $C_{p q}$ is the amplitude of order $(p, q)$ of the CSIG. A CSIG is thus a biperiodic array of pitch $a_{0}$. It can be considered as an $N$-wave interferometer, where $N$ is the number of orders selected. When illuminated at normal incidence by a monochromatic plane wave of complex amplitude $U_{0}$ and wavelength $\lambda$, a CSIG of transmittance $t(x, y)$ generates a field of complex amplitude $U(x, y, z)$ given by the general expression (see [18])

$$
\begin{aligned}
U(x, y, z)= & U_{0} \sum_{p, q} C_{p, q} \exp \left(\frac{2 i \pi}{\lambda} z \sqrt{1-\frac{\lambda^{2}\left(p^{2}+q^{2}\right)}{a_{0}^{2}}}\right) \\
& \times \exp \left(\frac{2 i \pi(p x+q y)}{a_{0}}\right),
\end{aligned}
$$

where $z$ is the direction normal to the plane of the CSIG.
Substituting Eqs. (3) and (4) into Eq. (5), we finally obtain the following expression for the field at normal incidence:

$$
U(x, y, z)=U_{0} \exp \left[\frac{2 i \pi}{\lambda} z \sqrt{1-\frac{\lambda^{2} \eta^{2}}{a_{0}^{2}}}\right] t(x, y) .
$$

We define the PSF of a CSIG as the intensity pattern $|U|^{2}$ observed in the detection plane at distance $z$. Its expression at normal incidence is given by

$$
\operatorname{PSF}(x, y)=|U(x, y, z)|^{2}=\left|U_{0}\right|^{2}|t(x, y)|^{2} .
$$

An important point highlighted by Eq. (7) is that at normal incidence, the intensity pattern of the field produced by this object is independent of $z$ in any plane normal to the $z$ axis. This property can be explained by the fact that the orders generated by the CSIG at normal incidence are always in phase, because they originate from a circle centered at the origin of the Fourier plane. Moreover, this object produces an achromatic self-image of its transmittance at any distance $z$. The PSFs at normal incidence of a 24-order CSIG and a 48-order CSIG are given in Fig. 2. When CSIGs are illuminated by a plane wave, they produce a field whose intensity profile is a propagation- and wavelength-invariant biperiodic array of bright spots, described by a $J_{0}$ function. The radius $r_{0}$ of the spots is given by the first zero of the $J_{0}$ function. In [14], it was shown that

$$
r_{0} \approx 0.38 a_{0} / \eta
$$

For imaging applications, a CSIG can thus be described by two parameters: the period $a_{0}$ of this pattern and $\eta$. The other characteristics can be derived from these two parameters.

\section{NONPARAXIAL EFFECTS IN OBLIQUE ILLUMINATION}

In oblique illumination, the $N$ orders diffracted by the CSIG are no longer in phase and Eq. (5) has to be modified in order to take into account these phase delays. Figure 3 illustrates a grating illuminated by an oblique ray. Parameters $\alpha_{1}$ and $\beta_{1}$ define the incident angle, and $\alpha_{2}$
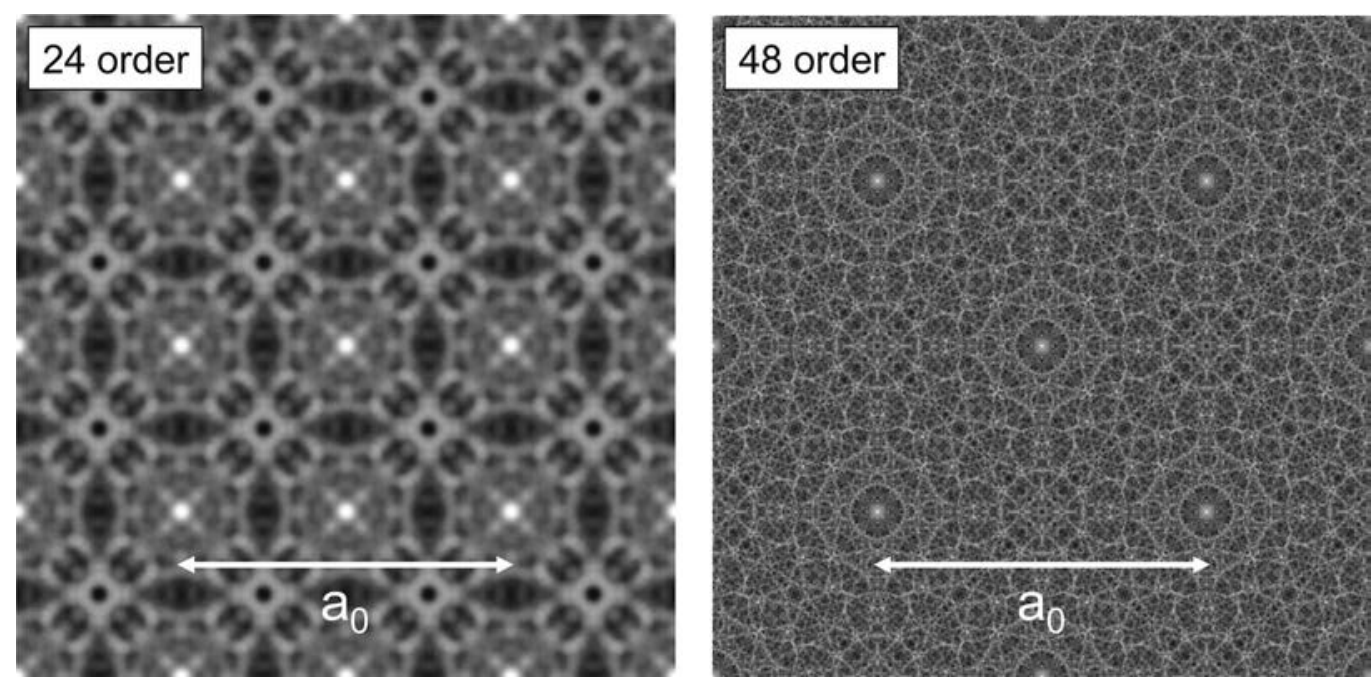

Fig. 2. PSFs of a 24-order CSIG $\left(\eta^{2}=650\right)$ and a 48 -order CSIG $\left(\eta^{2}=9425\right)$. 


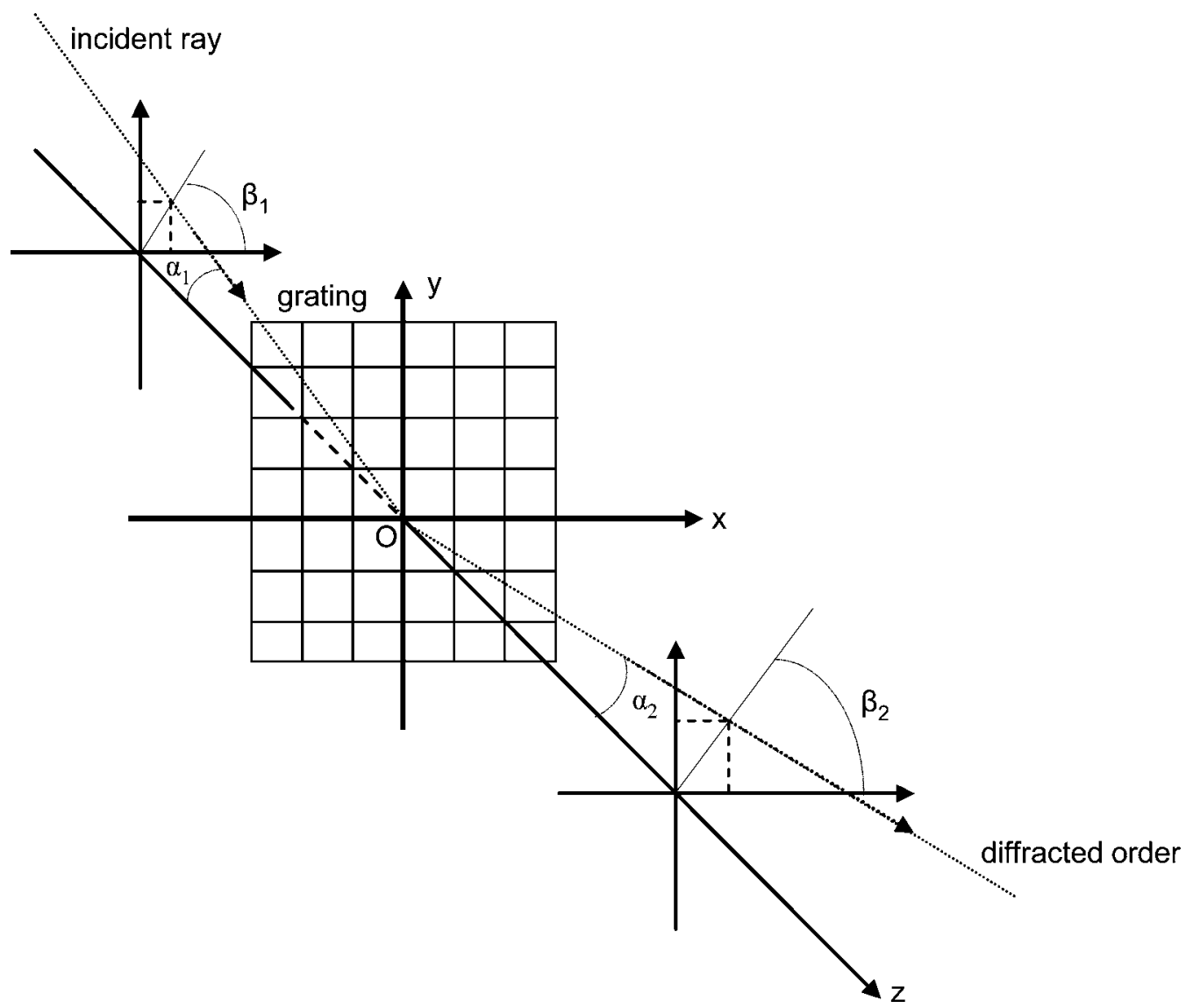

Fig. 3. Diffraction by a $2 \mathrm{D}$ periodic object.

and $\beta_{2}$ are the characteristics of one diffracted order. In order to simplify the following equations, we assume that $\beta_{1}=0$ so that rays coming through the grating are included in the $(x, z)$ plane. Using the approach of angular spectrum of plane waves (see [18]), Eq. (5) can be rewritten as a $2 \mathrm{D}$ Fourier series:

$$
\begin{aligned}
U(x, y, z)= & U_{0} \exp \left(\frac{2 i \pi}{\lambda} x \sin \left(\alpha_{1}\right)\right) \sum_{p, q} C_{p, q} \exp \left(i \phi_{p, q}(z)\right) \\
& \times \exp \left(\frac{2 i \pi(p x+q y)}{a_{0}}\right),
\end{aligned}
$$

where $\phi_{p, q}(z)$ are the phases of the orders generated by the CSIG in the detection plane at distance $z$ :

$$
\phi_{p, q}(z)=\frac{2 \pi}{\lambda} z \sqrt{1-\left(\sin \left(\alpha_{1}\right)+\frac{\lambda p}{a_{0}}\right)^{2}-\frac{\lambda^{2} q^{2}}{a_{0}^{2}}} .
$$

When $p \lambda / a_{0}$ and $q \lambda / a_{0}$ are small, the phases $\phi_{p, q}$ can be expanded in a Taylor series of $p$ and $q$ as follows: $\phi_{p, q}$ $=\phi_{p, q}^{(0)}+\phi_{p, q}^{(1)}+\phi_{p, q}^{(2)}+\phi_{p, q}^{(3)}+\phi_{p, q}^{(4)} \ldots$ Expansions up to order 4 are presented below:

$$
\begin{aligned}
\phi_{p q}^{(0)} & =\frac{2 \pi}{\lambda} \cos \left(\alpha_{1}\right) z, \\
\phi_{p q}^{(1)} & =-\frac{2 \pi}{\lambda} \frac{\lambda}{a_{0}} p \tan \left(\alpha_{1}\right) z,
\end{aligned}
$$

$$
\begin{aligned}
\phi_{p q}^{(2)}= & -\frac{2 \pi}{\lambda} \frac{1}{2} \frac{\lambda^{2}}{a_{0}^{2}}\left(\frac{p^{2}}{\cos ^{3}\left(\alpha_{1}\right)}+\frac{q^{2}}{\cos \left(\alpha_{1}\right)}\right) z \\
\phi_{p q}^{(3)}= & -\frac{2 \pi}{\lambda} \frac{1}{2} \frac{\lambda^{3}}{a_{0}^{3}}\left(\frac{\sin \left(\alpha_{1}\right) p^{3}}{\cos ^{5}\left(\alpha_{1}\right)}+\frac{\sin \left(\alpha_{1}\right) p q^{2}}{\cos ^{3}\left(\alpha_{1}\right)}\right) z \\
\phi_{p q}^{(4)}= & -\frac{2 \pi}{\lambda} \frac{1}{2} \frac{\lambda^{4}}{a_{0}^{4}}\left(\frac{4 \sin ^{2}\left(\alpha_{1}\right)+1}{4 \cos ^{7}\left(\alpha_{1}\right)} p^{4}+\frac{2 \sin ^{2}\left(\alpha_{1}\right)+1}{2 \cos ^{5}\left(\alpha_{1}\right)} p^{2} q^{2}\right. \\
& \left.+\frac{q^{4}}{4 \cos ^{3}\left(\alpha_{1}\right)}\right) z .
\end{aligned}
$$

This truncated Taylor series has already been expanded up to order 3 by Testorf et al. [19] for 1D periodic objects, that is, when $q=0$. In the case of CSIGs, all the terms $p$ and $q$ are linked to $\eta^{2}$ by relation (3). If we assume that $\alpha_{1}$ is small $\left[\cos \left(\alpha_{1}\right) \approx 1-\alpha_{1}^{2} / 2\right.$ and $\sin \left(\alpha_{1}\right) \approx \alpha_{1}$, the Taylor expansions can be simplified as follows by keeping the terms until the order 4:

$$
\begin{aligned}
& \phi_{p q}^{(1)}=-\frac{2 \pi}{\lambda} \frac{\lambda}{a_{0}}\left(p \alpha_{1}+\frac{1}{3} p \alpha_{1}^{3}\right) z \\
& \phi_{p q}^{(2)}=-\frac{2 \pi}{\lambda} \frac{1}{2} \frac{\lambda^{2}}{a_{0}^{2}}\left(\eta^{2}+\frac{1}{2} \eta^{2} \alpha_{1}^{2}+p^{2} \alpha_{1}^{2}\right) z,
\end{aligned}
$$




$$
\begin{aligned}
& \phi_{p q}^{(3)}=-\frac{2 \pi}{\lambda} \frac{1}{2} \frac{\lambda^{3}}{a_{0}^{3}} p \eta^{2} \alpha_{1} z, \\
& \phi_{p q}^{(4)}=-\frac{2 \pi}{\lambda} \frac{1}{8} \frac{\lambda^{4}}{a_{0}^{4}} \eta^{4} z .
\end{aligned}
$$

In order to link these developments to the formalism used for classical lenses, we define the wave aberration $W(\rho, \varphi: R)$ (see [20]) linked to the phase by the relation $\phi_{p q}=(2 \pi / \lambda) W(\rho, \varphi: R)[11,21]$. The usual expression of the wave aberration is

$$
\begin{aligned}
W(\rho, \varphi: R)= & \frac{1}{2} b_{1} \rho^{2}+b_{2} R \rho \cos (\varphi)+\frac{1}{4} c_{1} \rho^{4}+c_{2} R^{2} \rho^{2} \cos ^{2}(\varphi) \\
& +\frac{1}{2} c_{3} R^{2} \rho^{2}+c_{4} R^{3} \rho \cos (\varphi)+c_{5} R \rho^{3} \cos (\varphi),
\end{aligned}
$$

where the third, fourth, fifth, sixth, and seventh terms are third-order Seidel aberrations [21]. The first and second terms represent defocus and image shift, respectively. The third, fourth, fifth, sixth, and seventh terms indicate, respectively, third-order spherical aberration, astigmatism, curvature of field, distortion, and coma. The parameters $\rho, \varphi$, and $R$ are illustrated in Fig. 4; $R$ is the amplitude of the displacement of the PSF with respect to his position at normal incidence. Its relation is given by

$$
R=z \tan \left(\alpha_{1}\right),
$$

and $\rho$ and $\varphi$ are linked to the characteristics of the CSIG by the relations

$$
\begin{aligned}
& \frac{\rho \cos (\varphi)}{z}=\frac{\lambda p}{a_{0}}, \\
& \frac{\rho \sin (\varphi)}{z}=\frac{\lambda q}{a_{0}} .
\end{aligned}
$$

With these parameters, the various orders of the Taylor series of $\phi_{p q}$ become

$$
\phi_{p q}^{(1)}=-\frac{2 \pi}{\lambda} \frac{1}{z} R \rho \cos (\varphi)-\frac{2 \pi}{\lambda} \frac{1}{3 z^{3}} R^{3} \rho \cos (\varphi),
$$

$$
\begin{aligned}
\phi_{p q}^{(2)}= & -\frac{2 \pi}{\lambda} \frac{1}{2 z} \rho^{2}-\frac{2 \pi}{\lambda} \frac{1}{4 z^{3}} R^{2} \rho^{2} \\
& -\frac{2 \pi}{\lambda} \frac{1}{2 z^{3}} R^{2} \rho^{2} \cos ^{2}(\varphi), \\
\phi_{p q}^{(3)}= & -\frac{2 \pi}{\lambda} \frac{1}{2 z^{3}} R \rho^{3} \cos (\varphi), \\
\phi_{p q}^{(4)}= & -\frac{2 \pi}{\lambda} \frac{1}{8 z^{3}} \rho^{4} .
\end{aligned}
$$

Thanks to Eqs. (24)-(27), the expressions of the Seidel coefficients can be determined for a diffraction grating:

$$
\begin{aligned}
& b_{1}=b_{2}=-\frac{1}{z}, \\
& c_{1}=c_{2}=c_{3}=c_{5}=-\frac{1}{2 z^{3}}, \\
& c_{4}=-\frac{1}{3 z^{3}} .
\end{aligned}
$$

Thus, $\phi_{p q}^{(1)}$ contains the term of image shift and distortion; $\phi_{p q}^{(2)}$ the terms of defocus, astigmatism, and field curvature; $\phi_{p q}^{(3)}$ the terms of coma; and $\phi_{p q}^{(4)}$ the term of spherical aberration. In the case of a CSIG illuminated at $\lambda, \rho / z$ $=\lambda \eta / a_{0}=$ constant. Thus, as already demonstrated by Tanaka and Yamamoto [11], the intensity distribution of patterns created by those optical components are not affected by the coma aberration. Indeed, the aberration $(\rho / z)^{3} \cos (\varphi)$ behaves only as a tilt term of the wavefront and has no effect on the pattern except a shift. CSIGs also are not affected by spherical aberration, which plays the same role as the defocus, since its phase is constant. Finally, the aberrant phase $\phi_{\text {aberr }}$ that degrades the shape of the PSF is given by the terms of astigmatism, that is, the third term of $\phi_{p q}^{(2)}$ in Eq. (25):

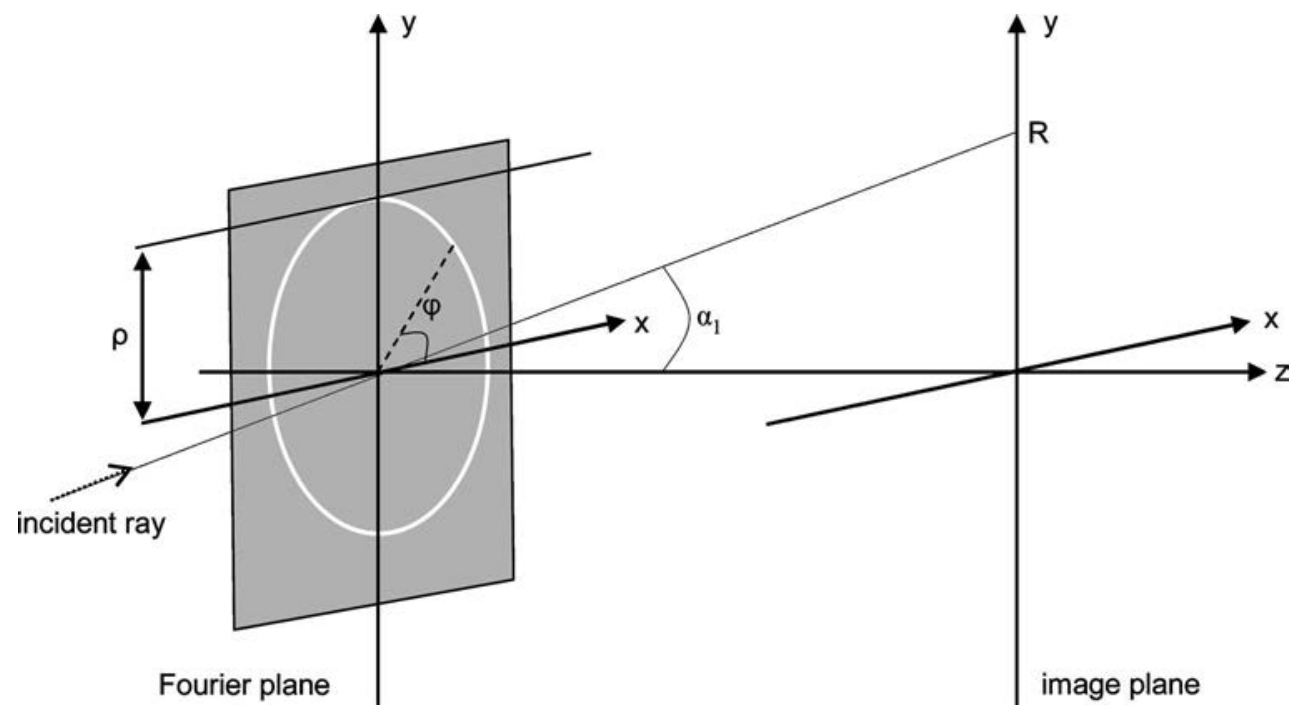

Fig. 4. Notation used to determine the expressions of the off-axis aberrations. 


$$
\phi_{\text {aberr }}(\rho, \varphi)=-\frac{2 \pi}{\lambda} \frac{1}{2 z^{3}} R^{2} \rho^{2} \cos (\varphi)^{2} .
$$

The PSF is obtained by taking the modulus square of Eq. (9) and can be written as follows:

$$
\begin{aligned}
P S F\left(x, y, \alpha_{1}\right)= & \left|U_{0}\right|^{2} \mid \sum_{p, q} C_{p, q} \exp \left[\frac{2 i \pi}{a_{0}}(p(x-\Delta x)+q y)\right] \\
& \times \exp \left[i \phi_{\text {aberr }}(p, q)\right] \mid
\end{aligned}
$$

where $\Delta x$ represents the shift of the pattern due to the effects of the tilt, the coma, aberration, and the distortion and whose expression is given by

$$
\Delta x=\left(1+\frac{1}{3} \alpha_{1}^{2}+\frac{1}{2} \frac{\lambda^{2}}{a_{0}^{2} \eta^{2}}\right) \alpha_{1} z .
$$

\section{CRITERIA FOR THE EVALUATION OF THE DEGRADATION OF THE PSF IN OBLIQUE ILLUMINATION}

\section{A. Peak-to-Valley Criterion}

The Peak-to-valley criterion can be used to estimate the tolerance angle $\alpha_{c}$ beyond which the spots generated by CSIGs are degraded by aberrations. The Peak-to-valley amplitude $\triangle P V$ is the amplitude between the biggest defect and the smallest defect on the aberrant phase $\phi_{\text {aberr }}$ of a wave; $\triangle P V$ is illustrated in Fig. 5 and is derived by the relation

$$
\Delta P V=\max \left(\phi_{\text {aberr }}\right)-\min \left(\phi_{\text {aberr }}\right)
$$

In the case of a small angle of incidence, the Peak-tovalley amplitude can be written as

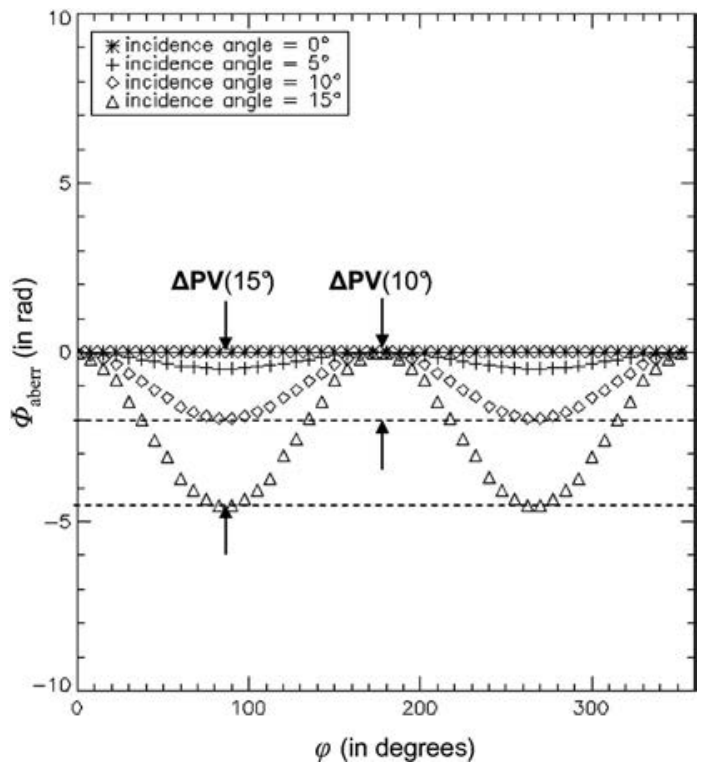

Fig. 5. Variation of the aberrant phase of a 48-order CSIG for different oblique illuminations.

$$
\Delta P V(\alpha)=\pi \frac{\lambda}{a_{0}^{2}} \eta^{2} \alpha^{2} z .
$$

The Rayleigh criterion (see [22]) postulates that the maximum phase delay that can be tolerated in an optical system is equal to $\pi / 2$. We can apply this criterion to the peak-to-valley amplitude in order to determine the tolerance angle $\alpha_{c}$ of a CSIG. The angle $\alpha_{c}$ is the maximal incidence angle of a beam in order to have a PSF not limited by astigmatism. A simple expression of $\alpha_{c}$ can be derived:

$$
\alpha_{c}=\frac{a_{0}}{\eta}\left(\frac{1}{2 \lambda z}\right)^{1 / 2} \approx 2 r_{0}\left(\frac{1}{\lambda z}\right)^{1 / 2},
$$

where $r_{0}$ is given by Eq. (8). The tolerance angle decreases when $r_{0}$ decreases and when the wavelength $\lambda$ increases. Parameters $r_{0}$ and $\lambda$ are thus limiting factors for the design of an illuminator with a high resolution (small $r_{0}$ ) and working at high wavelengths (e.g., in the infrared spectral range). Besides, the tolerance angle increases when the distance $z$ between the CSIG and the detection plane decreases. Miniaturization is thus advantageous for designing a wide-angle illuminator. We also realize that, when the parameters $z$ and $\lambda$ are fixed, there is a trade-off between a good tolerance in angles and a good resolution. In the case of two CSIGs at a distance $z=30 \mathrm{~mm}$ and at the wavelength $\lambda=4 \mu \mathrm{m}$, with the characteristics $\left[\eta^{2}\right.$ 650, $\left.a_{0}=1000 \mu \mathrm{m} ; r_{0}=15 \mu \mathrm{m}\right]$ and $\left[\eta^{2}=9425, \quad a_{0}\right.$ $\left.=7500 \mu \mathrm{m} ; r_{0}=30 \mu \mathrm{m}\right]$, the tolerance angles $\alpha_{c}$ are, respectively, equal to $4.5^{\circ}$ and $9^{\circ}$. These values are very close to those obtained by simulation and experimentation as we will see later on.

\section{B. RMS Criterion}

A finer criterion may be the root mean square $\Delta R M S$, which is calculated in the following way:

$$
\Delta R M S=\sqrt{\frac{\sum_{\varphi}\left(\phi_{\text {aberr }}(\varphi)-\bar{\phi}_{\text {aberr }}\right)^{2}}{N}},
$$

where $\bar{\phi}_{\text {aberr }}$ is the mean value of the aberrant phase and $\phi_{\text {aberr }}(\varphi)$ is the aberrant phase of the order placed at the angle $\varphi$. Notice that at a given distance $z$, the parameter $\rho$ is constant. The parameter $N$ is the number of orders generated by the CSIG. One advantage of the RMS criterion compared to the Peak-to-valley criterion is that in the former all the aberrant phase of the pupil plane is taken into account. The RMS also gives direct information on the image quality, which depends not only on the maximum deformation but also on the shape of the wavefront. We define the Strehl ratio in the case of CSIGs as the maximum intensity in a particular plane of observation normalized by the maximum intensity of an ideal CSIG not limited by off-axis aberrations:

$$
S=\frac{\max \left(P S F_{\alpha_{1}}\right)}{\max \left(P S F_{0^{\circ}}\right)} .
$$

When the aberrations are sufficiently small, the expression of the PSF given in Eq. (32) can be approximated as follows: 

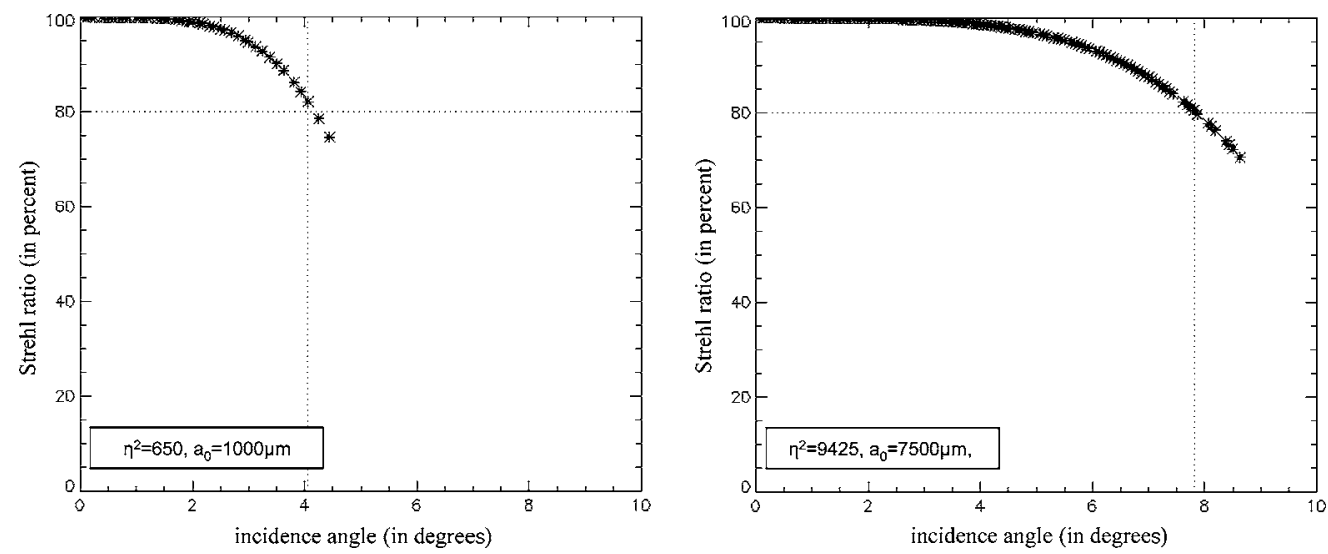

Fig. 6. Evolution of the Strehl ratio according to various incidence angles for two configurations of CSIGs.

$$
\begin{aligned}
P S F\left(x, y, \alpha_{1}\right)= & \left|U_{0}\right|^{2} \mid \sum_{p, q} C_{p, q} \exp \left[\frac{2 i \pi}{a_{0}}(p(x-\Delta x)+q y)\right] \\
& \times\left.\left(1+i \phi_{a b e r r}(p, q)-\frac{\phi_{a b e r r}^{2}(p, q)}{2}\right)\right|^{2} \\
\cong & \left|U_{0}\right|^{2}|t(x-\Delta x, y)|^{2}\left|1+i \bar{\phi}-\frac{\overline{\phi^{2}}}{2}\right|^{2} \\
\cong & P S F\left(x-\Delta x, y, 0^{\circ}\right)\left(1-\sigma_{\phi}^{2}\right),
\end{aligned}
$$

where $t(x, y)$ is the transmittance of the CSIG and $\bar{\phi}$ and $\sigma_{\phi}^{2}$ are, respectively, the average and the variance of the aberrant phase $\phi_{\text {aberr }}$. Equation (39) gives thus a simplified expression of the Strehl ratio. The Strehl ratio is linked to the RMS defined in Eq. (37) as follows:

$$
S \cong 1-\Delta R M S^{2} .
$$

Here we use the Marechal criterion [22], instead of the Rayleigh criterion used before. If the value of the RMS is greater than $\pi / 7$, then the optical component is limited by off-axis aberrations. The Marechal criterion corresponds to a Strehl ratio equal to $80 \%$. Figure 6 shows the evolution of Strehl ratios according to incidence angles and for different configurations of CSIGs. We use Eq. (40) to plot the graphs on Fig. 6, and we keep only the points greater than $70 \%$ in order to stay in the approximation of small aberration. In the case of two CSIGs at a distance $z=30 \mathrm{~mm}$ with the characteristics $\left[\eta^{2}=650, a_{0}=1000 \mu \mathrm{m}\right.$; $\left.r_{0}=15 \mu \mathrm{m}\right]$ and $\left[\eta^{2}=9425, a_{0}=7500 \mu \mathrm{m} ; r_{0}=30 \mu \mathrm{m}\right]$, the tolerance angles are, respectively, equal to $4^{\circ}$ and $7.8^{\circ}$. These values are close to the tolerance angles $\alpha_{c}$ established with the Rayleigh criterion.

\section{Definition of the Quality and Degradation Factors of a CSIG}

In this section, we want to find a criterion that conveys the degradation in intensity due to aberrations in the same way as the criteria that take into account the delay in phase of the different orders generated by a CSIG. This criterion will be used to estimate experimentally the tolerance angle $\alpha_{c}$ of CSIGs. Agreement between intensity degradation and phase delay about tolerance angle is expected. We define a quality factor $f$ by the relation

$$
f=\frac{1}{d^{2}} \frac{\int_{-d / 2}^{d / 2} \int_{-d / 2}^{d / 2}|I(x, y)|^{2} \mathrm{~d} x \mathrm{~d} y}{A V G^{2}},
$$

where $I$ represents the intensity of a square image of size $d$ and $A V G$ is the mean value of $I$ over an image and is given by the formula

$$
A V G=\frac{1}{d^{2}}\left|\int_{-d / 2}^{d / 2} \int_{-d / 2}^{d / 2} I(x, y) \mathrm{d} x \mathrm{~d} y\right| .
$$

We apply Eq. (41) to a biperiodic array of bright spots of diameter $\Delta$ and spaced by a period of $d$. The image takes the values equal to either 1 or 0 and is illustrated in Fig. 7. This configuration is close to the intensity pattern produced by a CSIG. The quality factor of this image can be expressed by the formula

$$
f=\frac{d^{2}}{\pi(\Delta / 2)^{2}} .
$$

This quality factor corresponds to the compression factor defined in [14] and is nearly equal to the factor $\eta^{2}(f$ $=1.1 \eta^{2}$ ). We also realize that the sharper the PSF, the big-

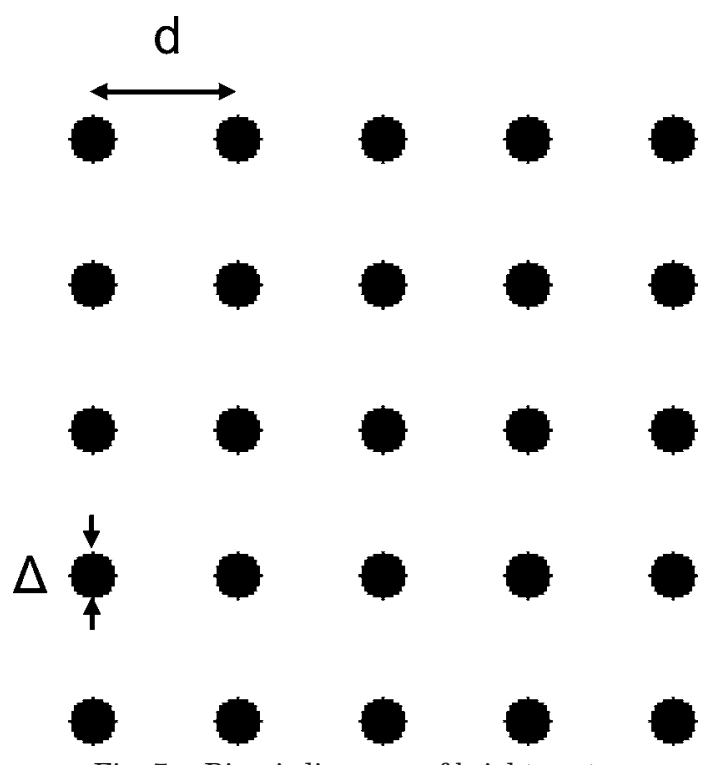

Fig. 7. Biperiodic array of bright spots. 


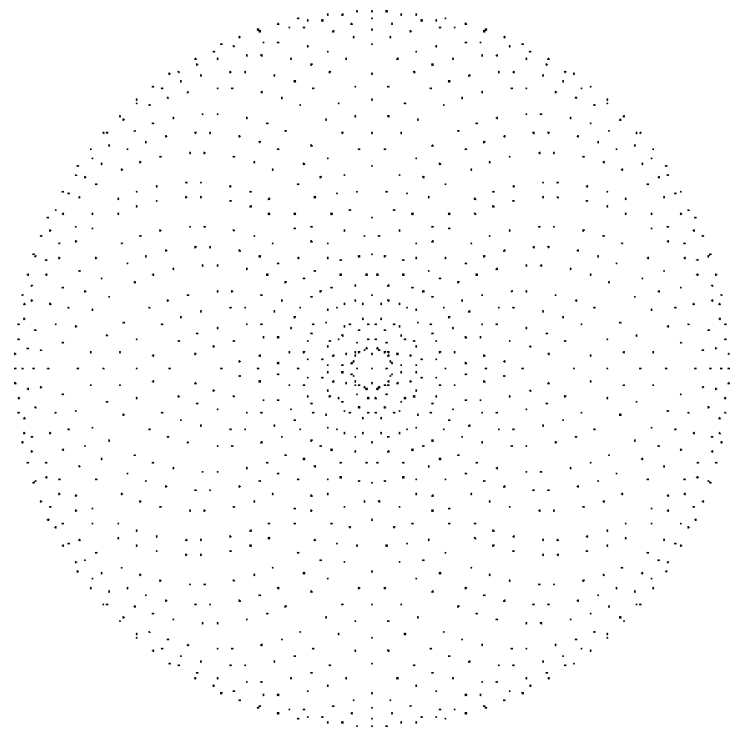

Fig. 8. PSF spectrum produced by a 48 -order CSIG $\left(\eta^{2}=9425\right)$ illuminated at normal incidence.

ger the quality factor. As off-axis aberrations are responsible for the widening of the PSF, they will also drop the quality factor. The quality factor can be expressed in the frequency domain [23], thanks to Parseval's theorem, by the relation

$$
f=\frac{\sum_{p q}\left|D_{p q}\right|^{2}}{D_{00}^{2}},
$$

where $D_{p q}$ designates the amplitudes of the various orders contained in the spectrum of the PSF pattern generated by the CSIG. Please note that $D_{p q}$ is different from $C_{p q}$ described before; indeed, $C_{p q}$ are the amplitudes of the various orders contained in the spectrum of the amplitude pattern generated by the CSIG. An example of the PSF spectrum is illustrated in Fig. 8. The orders $D_{p q_{(p, q) \neq 0}}$ give information about the repartition of light, whereas the order $D_{00}$ gives information about the global intensity of the wave. The amplitude of the order $D_{00}$ will also depend on the variation of the source intensity and will perturb the influence of the off-axis aberration on the degradation of the quality factor. To observe the degradation of the PSF linked to the off-axis aberrations, a degradation

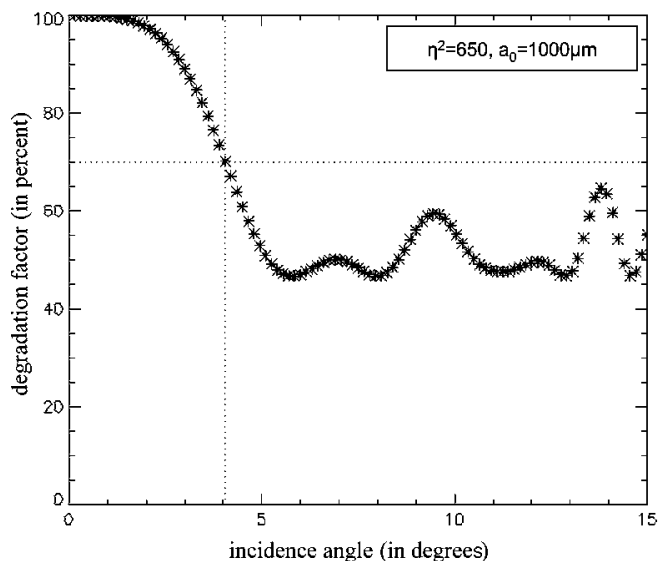

factor called $D$ is defined as follows:

$$
D=\frac{\left(\sum_{(p, q) \neq(0,0)}\left|D_{p q}\right|^{2}\right)_{\alpha_{1}}}{\left(\sum_{(p, q) \neq(0,0)}\left|D_{p q}\right|^{2}\right)_{0^{\circ}}} .
$$

The degradation factor $D$ compares the orders $D_{p q_{(p, q) \neq 0}}$ between the best case, at normal incidence, and a case in oblique illumination. This factor is dimensionless and does not depend on the variation of the source intensity. Figure 9 gives the degradation factor for various configurations of CSIGs. In the case of two CSIGs at a distance $z=30 \mathrm{~mm}$ with the characteristics $\left[\eta^{2}=650, a_{0}=1000 \mu \mathrm{m}\right]$ and $\left[\eta^{2}=9425, a_{0}=7500 \mu \mathrm{m}\right]$, a criterion of $70 \%$ for the degradation factor gives the same tolerance angles determined with the classical Strehl ratio criterion (80\%). The two CSIGs have, respectively, a tolerance angle of $4^{\circ}$ and $7.8^{\circ}$

\section{EXPERIMENTAL STUDY}

\section{A. Description of the Setup}

Figure 10 displays the experimental setup for observing the intensity distribution of the PSF created by a CSIG at various incident angles. A mask is illuminated by a blackbody at $1000 \mathrm{~K}$ and is collimated by a collimator of focal length equal to $760 \mathrm{~mm}$. The scene generated is imaged by a CSIG placed at a variable distance from an infrared focal plane array (detection spectral range of [3-5.5 $\mu \mathrm{m}]$ ). The complementary metal oxide semiconductor is composed of $384 \times 288$ pixels of $20 \mu \mathrm{m}$ in size. The camera and the grating are mounted on a rotation stage so that the scene generated can be seen through various incident angles. As we want to study the PSF of a CSIG, we generate a point source by using a pinhole of diameter $1 \mathrm{~mm}$ or $2 \mathrm{~mm}$. We studied experimentally a CSIG with the characteristics $\left[\eta^{2}=650, a_{0}=1000 \mu \mathrm{m}\right]$ and placed at a distance of $30 \mathrm{~mm}$ from the detector. The grating used for this experiment is a two-level approximation of the ideal transmittance as described in [15]. It is a binary-phase grating on a GaAs substrate.

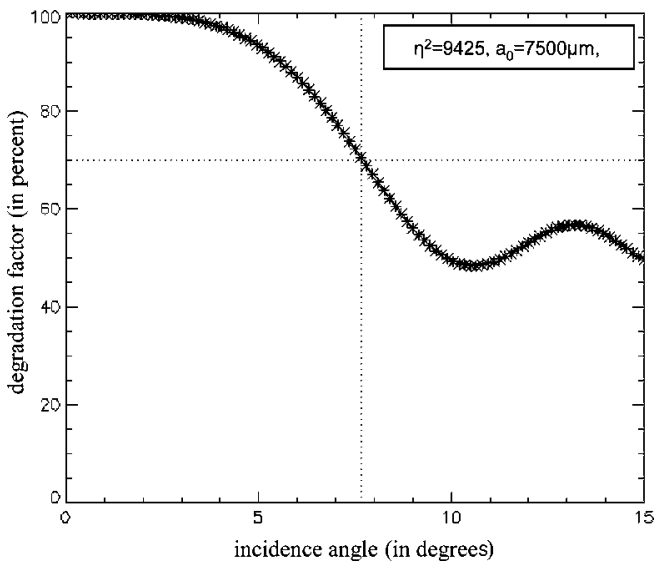

Fig. 9. Theoretical evolution of the degradation factor $D$ versus incidence angle $\alpha_{1}$ for two different configurations of CSIGs. 


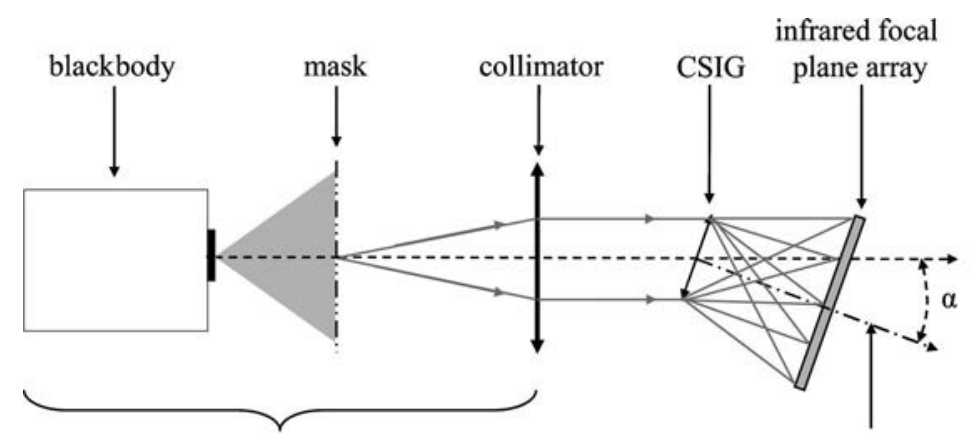

infrared scene generation

optical axis of the system

Fig. 10. Presentation of the experimental setup.
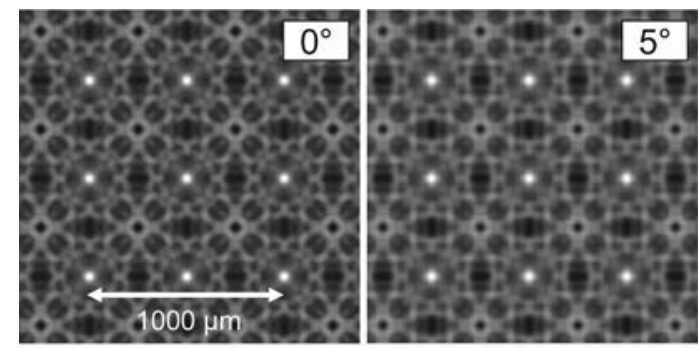

(a)
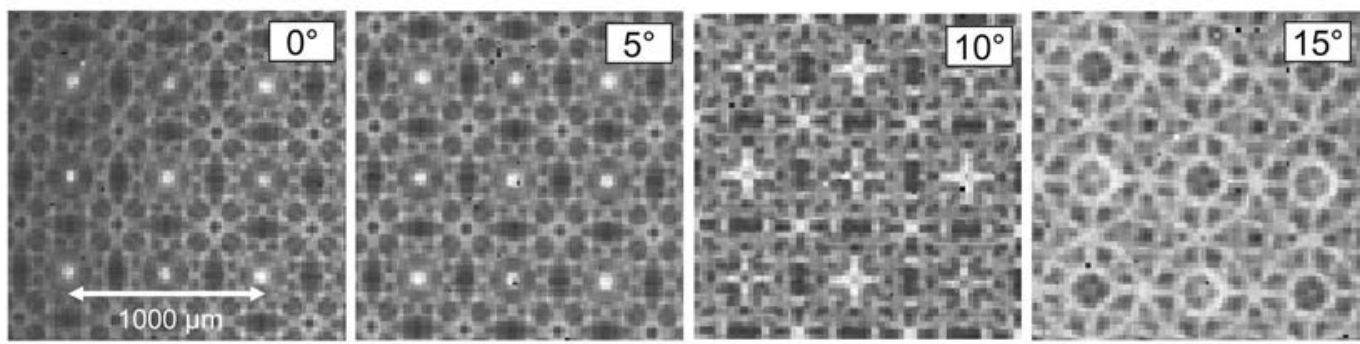

(b)

Fig. 11. PSF of a 24-order CSIG $\left(\eta^{2}=650\right)$ obtained by either simulation (a) or experimentation (b) for various incidence angles.

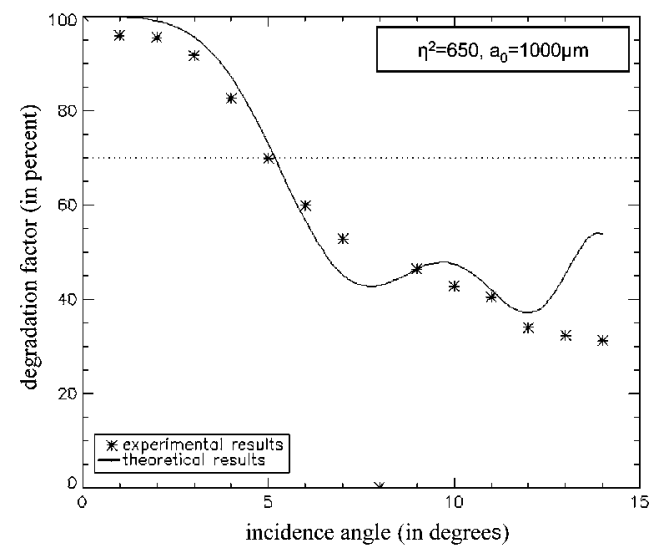

(a)

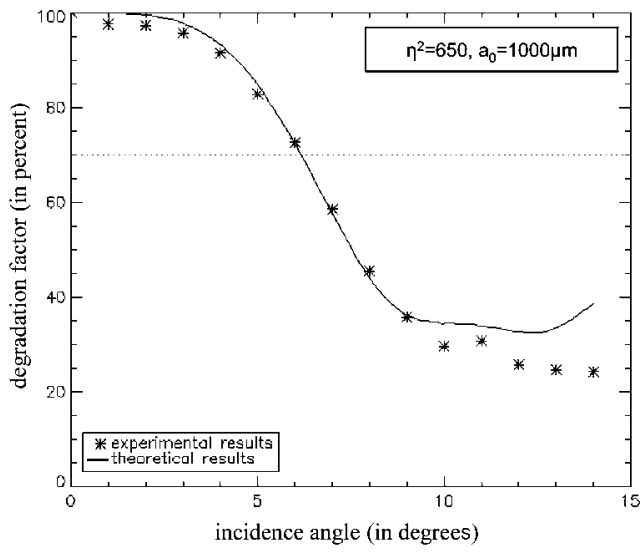

(b)

Fig. 12. Comparison of the degradation factor obtained by simulation and experimentation in the case of pinhole masks with a diameter of (a) $1 \mathrm{~mm}$ or (b) $2 \mathrm{~mm}$.

\section{B. Confrontation of Measures Obtained by Simulation and Experimentation}

The PSFs of these CSIGs for various incident angles are compared with those computed, using Eq. (9). Figure 11 shows some experimental and simulated results created by oblique plane-wave illumination. The incident angles onto the CSIG are $\theta=0^{\circ}, 5^{\circ}, 10^{\circ}$, and $15^{\circ}$. We notice that the PSFs observed experimentally agree fairly well with 
those obtained numerically. We determine the degradation factor of the 24-order CSIG with the PSFs measured experimentally. This degradation factor is compared in Fig. 12 with the one obtained by simulation for different sizes of pinhole mask. In the simulation, we take into account the influence of the pixel size and the influence of the diameter of the pinhole masks used for this experiment. Both attenuate the small degradations of the shape of the PSF and increase the value of the cutoff angle. Fairly good agreements between the experiment and the simulation are observed.

\section{CONCLUSION}

In this paper, we have determined the expressions of the third-order aberrations that affect the quality of the PSF of a 2D diffraction grating, and, to our knowledge, the expressions of the Seidel coefficients for a field generated by a diffraction grating have never been established before. This formalism has been applied to CSIGs. It turns out that the shape of the PSF of a CSIG is degraded in angle by the aberration of astigmatism, whereas the coma aberration shifts only the PSF. Different criteria on the phase delay of the orders generated by CSIGs have been established to assess the tolerance angle of the CSIGs. We have used the Rayleigh criterion on the peak-to-valley relation and the Marechal criterion on the RMS relation (or, equivalently, the Strehl ratio) to determine the tolerance angles of various configurations of CSIGs. These two methods give similar results. The Rayleigh approach establishes an engineer rule that links the tolerance angle to the resolution and the compactness of the optical system. It shows that a well-designed CSIG can have a satisfactory tolerance in angle. A compromise between tolerance in angle and resolution has, however, to be found, depending on application. Nevertheless, this compromise is not severely restricting for designing unconventional imaging systems based on CSIGs with a sufficient field of view and a long depth of focus. The theoretical study and the simulations have been illustrated by experiments. A degradation factor has been established for this occasion in order to extract a tolerance angle from the experimental PSFs, and fairly good agreement between what we predicted and what we observed has been reported. The formalism and the results presented in this paper can be easily generalized to other Cartesian or circular gratings (e.g., diffractive axicons) and can be useful to study their imaging properties in the case of a more complex scene with a given field of view.

\section{ACKNOWLEDGMENT}

This work was sponsored by the Délégation Générale de l'Armement (DGA) of the French Ministry of Defense.

\section{REFERENCES}

1. J. Durnin, "Exact solutions for nondiffracting beams. I. The scalar theory," J. Opt. Soc. Am. 4, 651-654 (1987).

2. J. H. McLoed, "The axicon: a new type of optical element," J. Opt. Soc. Am. 14, 592-597 (1954).

3. L. M. Soroko, "Axicons and meso-optical imaging devices," in Progress in Optics, Vol. 27, E. Wolf, ed. (Elsevier, 1989), pp. 111-127.

4. J. Sochacki, A. Kolodziejczyk, Z. Jaroszewicz, and S. Bara "Nonparaxial design of generalized axicons," Appl. Opt. 31, 5326-5330 (1992).

5. A. T. Friberg, "Stationary-phase analysis of generalized axicons," J. Opt. Soc. Am. A 13, 743-750 (1996).

6. Z. Jaroszewicz and J. Morales, "Lens axicons: systems composed of a diverging aberrated lens and a perfect converging lens," J. Opt. Soc. Am. A 15, 2383-2390 (1998).

7. C. J. Zapata-Rodríguez and A. Sánchez-Losa, "Threedimensional field distribution in the focal region of lowFresnel-number axicons," J. Opt. Soc. Am. A 23, 3016-3026 (2006).

8. R. Arimoto, C. Saloma, T. Tanaka, and S. Kawata, "Imaging properties of axicon in a scanning optical system," Appl. Opt. 31, 6653-6657 (1992).

9. T. Grosjean, F. Baida, and D. Courjon, "Conical optics: the solution to confine light," Appl. Opt. 46, 1994-2000 (2007).

10. Z. Bin and L. Zhu, "Diffraction property of an axicon in oblique illumination,” Appl. Opt. 37, 2563-2568 (1998).

11. T. Tanaka and S. Yamamoto, "Comparison of aberration between axicon and lens," Opt. Commun. 184, 113-118 (2000).

12. A. Thaning, Z. Jaroszewicz, and A. T. Friberg, "Diffractive axicons in oblique illumination: analysis and experiments and comparaison with elliptical axicons," Appl. Opt. 42, 9-17 (2003).

13. A. Burvall, K. Kolacz, A. V. Goncharov, Z. Jaroszewicz, and C. Dainty, "Lens axicons in oblique illumination," Appl. Opt. 46, 312-318 (2007).

14. N. Guérineau and J. Primot, "Nondiffracting array generation using an $N$-wave interferometer," J. Opt. Soc. Am. 16, 293-298 (1999).

15. N. Guérineau, B. Harchaoui, J. Primot, and K. Heggarty, "Generation of achromatic and propagation-invariant spot arrays by use of continuously self-imaging gratings," Opt. Lett. 26, 411-413 (2001).

16. J. Primot and N. Guérineau, "Extended Hartmann test based on the pseudoguiding property of a Hartmann mask completed by a phase chessboard," Appl. Opt. 39, 5715-5720 (2000).

17. N. Guérineau, S. Rommeluere, E. Di Mambro, I. Ribet, and J. Primot, "New techniques of characterization," C. R. Phys. 4, 1175-1185 (2003).

18. R. F. Edgar, "The Fresnel diffraction images of periodic structures," Opt. Acta 16, 281-287 (1969).

19. M. Testorf, J. Jahns, N. A. Khilo, and A. M. Goncharenko "Talbot effect for oblique angle of light propagation," Opt. Commun. 129, 167-172 (1996).

20. J. W. Goodman, Introduction to Fourier Optics (McGrawHill, 1968), p. 101.

21. M. Born and E. Wolf, Principles of Optics, 6th ed. (Pergamon, 1989), Chap. V, p. 203.

22. M. Born and E. Wolf, Principles of Optics, 6th ed. (Pergamon, 1989), Chap. IX, p. 468.

23. E. di Mambro, R. Haïdar, N. Guérineau, and J. Primot, "Sharpness limitations in the projection of thin lines by use of the Talbot experiment," J. Opt. Soc. Am. A 21, 2276-2282 (2004). 